\title{
Article \\ An Innovative Infrastructure Based on Shape-Adaptive RIS for Smart Industrial IoTs
}

\author{
Jiazheng Wang ${ }^{1}$, Xiuhua Fu ${ }^{2,3, *}$, Rongqun Peng ${ }^{2,3}$ and Michel Kadoch ${ }^{4}$ \\ 1 School of Physics and Optoelectronic Engineering, Shandong University of Technology, Zibo 255000, China; \\ jzhwang@sdut.edu.cn \\ 2 School of Computer Science and Technology, Shandong University of Technology, Zibo 255000, China; \\ pengrq@sdut.edu.cn \\ 3 State Key Laboratory of Networking and Switching Technology, \\ Beijing University of Posts and Telecommunications, Beijing 100876, China \\ 4 Department of Electrical Engineering, ETS, University of Quebec, Montreal, QC H3C 3J7, Canada; \\ michel.kadoch@etsmtl.ca \\ * Correspondence: xhfu@sdut.edu.cn
}

check for updates

Citation: Wang, J.; Fu, X.; Peng, R.; Kadoch, M. An Innovative Infrastructure Based on ShapeAdaptive RIS for Smart Industrial IoTs. Electronics 2022, 11, 391. https://doi.org/10.3390/electronics 11030391

Academic Editor: Rashid Mehmood

Received: 26 November 2021

Accepted: 25 January 2022

Published: 27 January 2022

Publisher's Note: MDPI stays neutral with regard to jurisdictional claims in published maps and institutional affiliations.

Copyright: (C) 2022 by the authors. Licensee MDPI, Basel, Switzerland. This article is an open access article distributed under the terms and conditions of the Creative Commons Attribution (CC BY) license (https:// creativecommons.org/licenses/by/ $4.0 /)$.

\begin{abstract}
Industrial Internet of Things (IoTs) applications will have higher demands in terms of system capacity and wireless transmission performance in the future. Reconfigurable intelligent surfaces (RIS) for the wireless transmission environment reconstruction is currently a research hotspot in the field of wireless communication. In this paper, RIS is applied to industrial IoTs scenarios, and an innovative infrastructure based on shape-adaptive RIS suitable for smart industrial IoTs is proposed. Based on the many advantages of RIS, the proposed infrastructure can meet the significant demands of industrial IoTs in terms of network coverage, system capacity, transmission rate, and communication security. Further, in order to achieve flexible RIS phase adjustment in wider ranges, in this paper, a shape-adaptive RIS mechanism is proposed to regulate RIS-reflected waves in the shape domain, which can be achieved by scalable RIS unit sizes and macroscopically changeable physical shapes. This mechanism can reduce the requirements for RIS-controller and artificial-unit design and expand the spatial distribution of reflected beams, whereas this is difficult to achieve by conventional regulations in the spatial, time, and frequency domains. The simulation results show that, in terms of system-coverage probability, the system performance of RIS is better than that of general communication systems, and the performance of the deformable RIS-based system is the best. In terms of channel gain, the performance of the deformable RIS is related to its surface size. Moreover, the path loss caused by the long-distance transmission of wireless signals can be compensated for by increasing the RIS surface area.
\end{abstract}

Keywords: reconfigurable intelligent surfaces (RIS); shape-adaptive RIS; industrial Internet of Things (IoTs); 5G/6G; channel gain

\section{Introduction}

With the development and maturity of 5G and the Internet of Things (IoTs), the global digital revolution is experiencing a new round of industrial transformation. The integrated development of 5G and the industrial IoTs has woven a series of visions of 5G-based smart industrial applications [1]. VR/AR, ultra-high-definition video, machine vision, industrial robots, unmanned aerial vehicles (UAVs), and remote control are typical applications of the smart industrial IoTs. These applications have ultra-high requirements for bandwidth, connectivity, coverage, latency, and other aspects. For example, in smartfactory applications, users need to install a variety of sensors and cameras in the factory so that they can monitor any equipment and workflow in real time [2]. The wireless network signal should be able to cover any location in the factory area to ensure that the sensor 
and camera data can be transmitted to the network infrastructure with high quality and at high speeds.

In recent years, many large companies around the world have been building a new type of smart factory called a "dark factory". In China, these companies include Alibaba, GREE Electric Appliances Inc., Xiaomi Inc., etc. GREE is striving to build a fully automatic, intelligent, and 5G-covered "dark factory", which will only need 80,000 people to achieve an output value of RMB 200 billion, much higher than the current output value of RMB 80 billion created by more than 100,000 people. Xiaomi has invested RMB 600 million to build its own smart factory, hoping to complete the entire production process with robots in the future. Smart factory or dark factory refers to a kind of production environment in which industrial robots use artificial intelligence technology for information processing and inference prediction, thus realizing a fully automated industrial product-design and manufacturing process without human involvement. Figure 1 depicts the production process of smart factories aided by wireless networks. Smart factories will be a major feature in the era of Industry 5.0 [3-5].

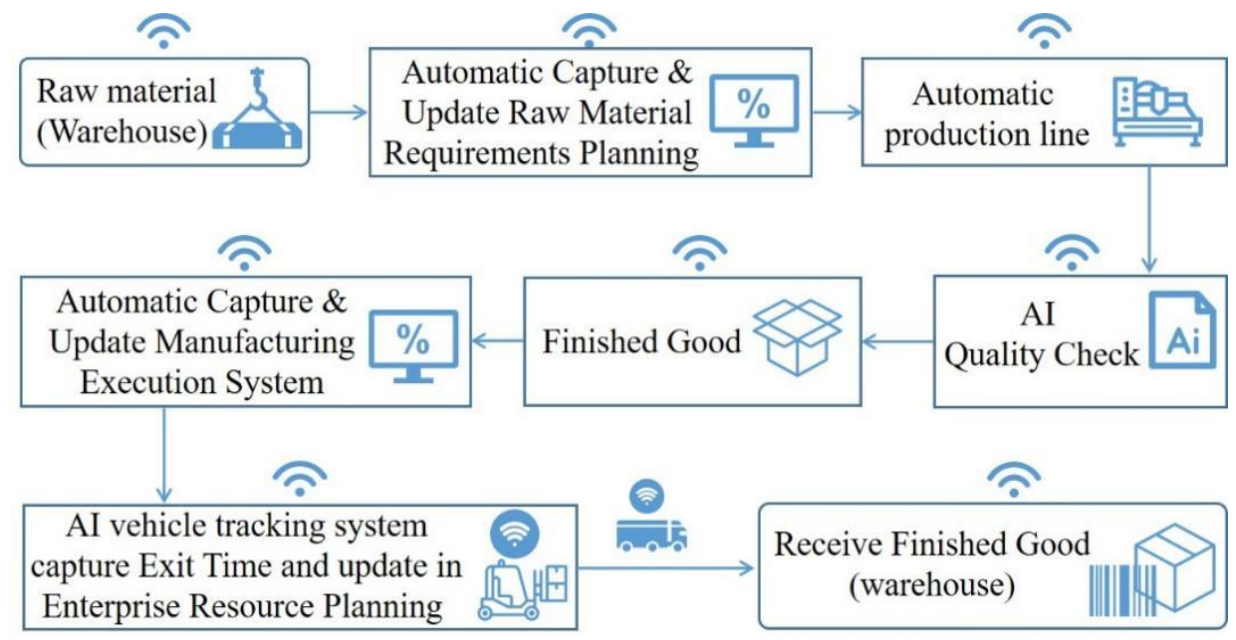

Figure 1. The smart-factory production process aided by wireless networks.

The foundation of the intelligent industrial IoTs consists of building communicationnetwork infrastructure. The 5G network currently under construction mainly works in the sub-6G frequency band, which cannot meet the ultra-high demands of the future industrial Internet. Fortunately, B5G (working in the millimeter wave band) and 6G mobile networks (working in the $\mathrm{THz}$ band) are being developed towards a unified network that connects virtual and real space and realizes ubiquitous intelligent connection. One of the missions of $6 \mathrm{G}$ is to provide QoS-guaranteed secure connections for industrial applications [6]. However, the propagation distances of millimeter waves and $\mathrm{THz}$ radio waves are relatively short, their diffraction and transmission abilities are very poor, and they are easily blocked by obstacles [7]. Therefore, in typical industrial IoTs scenarios, such as workshops and factories, radio-signal coverage and signal transmission are critical issues that determine whether the future mobile network can successfully integrate with the industrial Internet.

Reconfigurable intelligent surfaces (RIS) for the reconstruction of the wireless transmission environment is a research hotspot in the field of wireless communication in recent years. RIS is an artificial electromagnetic metamaterial with digitally programmable properties, the surface of which consists of a large number of low-cost, artificially designed reflective units. With an intelligent controller, RIS can realize the reflection of incident waves in different amplitudes, phases, and polarization directions. Due to its beamforming capabilities, RIS offers significant advantages in enhancing network coverage and increasing data-transmission rates. 
RIS requires collaborative research across disciplines, including wireless communications, RF engineering, electromagnetics, and metamaterials. Current RIS research is focused on three areas. The first research area is the design of RIS hardware structures, as well as functional implementation and performance measurement based on prototype systems. Research in this area specifically includes the design of RIS artificial units and their spatial arrangement, the production of adjustable materials or electronic components of the units, and the realization of RIS control mechanisms for different purposes. The second area is the theoretical study of baseband algorithms based on mathematical models. Studies in this area are very rich, including channel modeling, channel estimation, beamforming, simultaneous wireless information and power transfer (SWIPT) technology, secure communication, and so on. The third area is the design of RIS-based wireless network architecture. Most research in this area is aimed at specific applications to meet the requirements of specific scenarios. Table 1 shows the main research studies of RIS in different areas, as well as their main features.

Table 1. Comparison of different RIS studies.

\begin{tabular}{|c|c|c|c|}
\hline Classification & References & Features & Technology Trends \\
\hline $\begin{array}{l}\text { Design of RIS hardware } \\
\text { structures }\end{array}$ & [8-13] & $\begin{array}{l}\text { Reflective type, emission type, penetrable type, pin } \\
\text { diode, MEMS, FPGA, amplitude, phase, frequency, } \\
\text { polarization, OAM, metasurface with planar array. }\end{array}$ & $\begin{array}{c}\text { Independently controllable units, adjustable in } \\
\text { large bandwidth, optimization of spatial } \\
\text { arrangement, versatile RIS array. }\end{array}$ \\
\hline $\begin{array}{l}\text { Theoretical study of } \\
\text { baseband algorithms }\end{array}$ & [14-19] & $\begin{array}{l}\text { Passive, active, cascaded channel, phase-shift matrix, } \\
\text { channel modeling, channel estimation, channel state } \\
\text { information (CSI), signal enhancement, signal neutralization, } \\
\text { SWIPT, communication perception and localization. }\end{array}$ & $\begin{array}{l}\text { Different transmission scenarios, distributed } \\
\text { RIS collaborative-transport solutions, AI-based } \\
\text { algorithms, system-level simulation platforms. }\end{array}$ \\
\hline \multirow{2}{*}{$\begin{array}{l}\text { Design of RIS-based } \\
\text { wireless network } \\
\text { architecture }\end{array}$} & [20-24] & $\begin{array}{l}\text { Single RIS; multi-RIS collaboration; based on spectrum } \\
\text { sharing; based on non-spectrum sharing; 3D } \\
\text { beamforming; combination with different networks, } \\
\text { such as MIMO, OFDM. }\end{array}$ & \multirow{2}{*}{$\begin{array}{l}\text { Topology of multi-cell RIS networks, mobility, } \\
\text { security, deployment, applications. Industrial } \\
\text { IoT is expected to be a major application scenario } \\
\text { for RIS in the future, and RIS will aid in the } \\
\text { realization of various new applications in the } \\
\text { industrial IoTs, such as digital twins (DTs). }\end{array}$} \\
\hline & this study & $\begin{array}{l}\text { Industrial IoTs, IoTs applications, shape-adaptive RIS, } \\
\text { infrastructure, coverage probability, channel gain. }\end{array}$ & \\
\hline
\end{tabular}

Table 1 shows that the vast majority of RIS studies are focused on the first and second research areas, while only a small number of studies is concentrated on RIS-based networks and applications. This study is dedicated to providing RIS-based wireless-transmission support for future industrial IoTs applications. Based on its many advantages, RIS is well suited to meet the significant demands of the industrial IoTs in terms of network coverage, system capacity, data-transmission rate, communication security, etc.

Undoubtedly, the large-scale application of RIS will be closely related to the difficulty of RIS deployment. RIS is generally deployed on the surface of various objects, which requires the surface shape of RIS to be well adapted to that of the objects. However, current RIS generally has a two-dimensional planar surface, and this fixed structure has certain limitations in terms of deployment and functional implementation. Therefore, this paper further investigates the RIS metasurface structure with the aim of making the RIS surface shape adaptive to that of the deployed objects.

An innovative infrastructure for the smart industrial IoTs is proposed, which uses 5G/B5G/6G base stations to provide the main coverage and intelligent edge-computing deployed at the edge of the network to provide intelligent interconnection, intelligent computing, intelligent control, etc. The innovation of this architecture lies in the deployment of a large number of low-cost, reconfigurable intelligent surfaces (RIS) at suitable locations in a given industrial IoTs scenario, as well as the fact that the shape of RIS can be adaptively changed. The intelligent-reflection and signal-enhancement capabilities of RIS can be used to achieve comprehensive network coverage without blind spots in various industrial IoTs scenarios and greatly increase the data-transmission rate. Moreover, by using the signal-neutralization capability of RIS, secure communications can also be implemented in the physical layer of RIS-assisted industrial IoTs applications [25]. 
The structure of this paper is as follows. Section 2 introduces the structure of RIS and its communication capabilities when combined with wireless communication networks. In Section 3, we propose an innovative infrastructure based on shape-adaptive RIS suitable for $5 \mathrm{G}$ intelligent industrial IoTs applications, and the composition and function of the network infrastructure are described. Further, the design of shape-adaptive RIS is described in Section 4. Simulation and performance analyses of shape-adaptive RIS are carried out in Section 5. Section 6 summarizes the full text.

\section{What Is RIS?}

\subsection{Structure and Artificial Units of RIS}

RIS is an advanced digital metamaterial that can control the electromagnetic properties of the metamaterial units in real time through the input a digital coding sequence, thereby realizing real-time control of electromagnetic wave functions. Consistent with binary computer technology, digital metamaterials realize different electromagnetic wave functions in the physical world through binary-digit sequences in the digital world, thereby establishing a connection between the physical world and the digital world. Digital metamaterials are also called informational metamaterials because different digital coding sequences can represent different information. Cui et al. [8] proposed the concept of digitally coded information metamaterials. They used binary 0 and 1 to represent metamaterial artificial units that can encode all artificial units in binary space in real time, thus realizing the first digital information metamaterial.

The design principle of RIS is to implement multiple basic circuit units (artificial units) according to a certain rule (usually distributed in a regular array) on the entire metamaterial surface. The electromagnetic characteristics of each artificial unit are digitally controllable. By inputting different binary control sequences, real-time adjustment of the amplitude, phase, frequency, and other parameters of the incident electromagnetic wave on each artificial unit is realized so that RIS show different physical phenomena on the whole and express different information or achieve certain application purposes.

The structure of RIS is shown in Figure 2. In terms of hardware structure, RIS mainly include three parts. The first layer is the metamaterial surface that is in direct contact with the outside world. It is composed of a large number of low-cost artificial units. Electromagnetic waves are incident on the surface, and under the overall electromagnetic response presented by all the reflection units, different numbers of beams or beams pointed in different directions are formed. The second layer is a metal back plate with a certain structure, which is used to avoid the leakage of incident electromagnetic waves behind the metamaterial and improve the reflection efficiency. The third layer is the control circuit board, which is used to control the state of artificial units in real time, realize real-time control of incident waves, and achieve different reflection effects. This is the core of RIS, acting as an intelligent controller. In the current implementation, the control circuit is usually implemented by FPGA [18]. The FPGA circuit generates different binary sequences and sends them to the artificial unit. By changing the state or electromagnetic characteristics of the artificial unit, real-time control of incident waves is realized. Therefore, by controlling the output signal of the FPGA, different physical realizations, such as single-beam radiation, multi-beam radiation, and beam scanning, can be realized, thereby establishing a connection between the digital world and the physical world. 


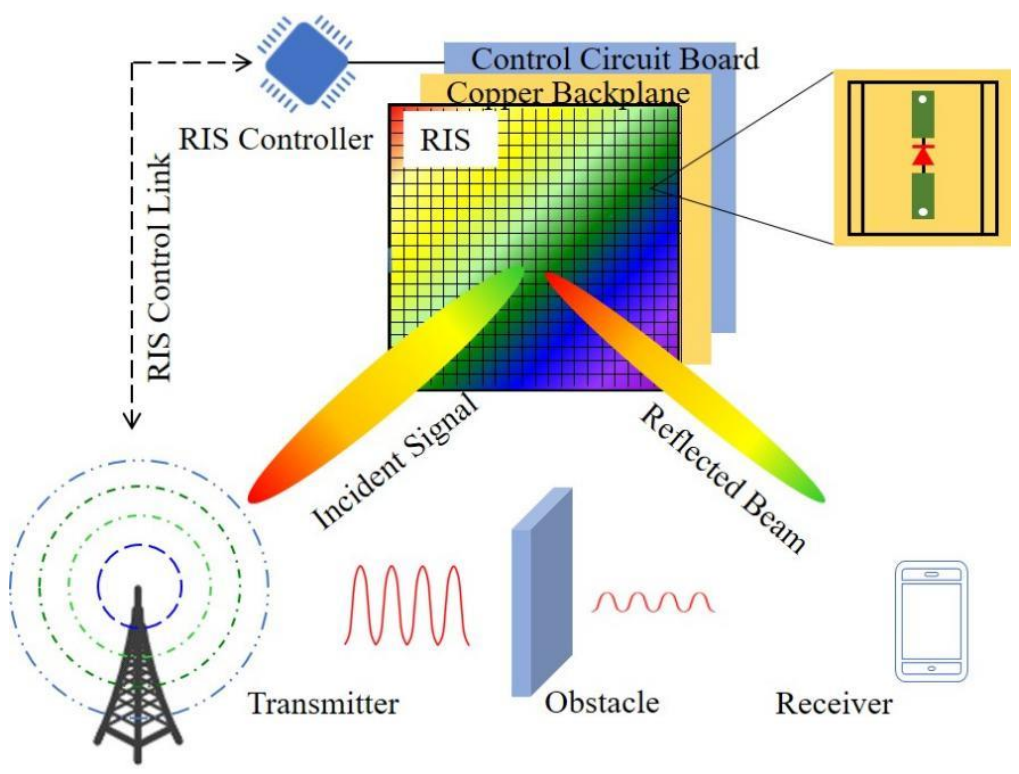

Figure 2. The structure of RIS.

In terms of physical units, the basic circuit of the artificial unit should be able to present at least two different states, such as on and off, and be able to switch between different states flexibly under the action of an external excitation signal. At the same time, considering the implementation cost of metamaterials composed of a large number of artificial units, the cost of the electronic devices used in these artificial units is also important. Of course, the state accuracy of these devices should be fully guaranteed. At present, in most cases, the artificial unit is composed of a diode (PIN tube) in the laboratory, and the PIN tube is controlled by an external signal to make the artificial unit present in two different states. For example, when different voltage signals (high voltage represents 1; low voltage represents 0 ) are fed into the artificial unit, the basic circuit composed of the PIN tube will be on or off, which causes the circuit to form one of two different electromagnetic responses. Of course, artificial units can also be realized by other devices, such as triodes, MEMES, graphene, thermosensitive devices, photosensitive devices, etc. [26,27].

Since RIS can generate varying beams of incident waves and can realize beam shaping, they can be regarded as an antenna. Each artificial unit of RIS is actually a tiny antenna, and each is independent, only reflecting the signal incident on itself, according to its electromagnetic characteristics. All artificial units form a miniature antenna array according to the physical structure, which presents a tightly coupled antenna as a whole. Similarly to traditional antenna size requirements, in order to better propagate electromagnetic waves, each artificial unit is very small-about $1 / 10 \sim 1 / 4$ of the working wavelength, called subwavelength size - and the units are closely spaced. Theoretically, the state of each artificial unit is independent and controllable. In practice, all coding programs can be written into FPGA, and it supports field-programmable, real-time control of different functions of RIS and realizes free switching among single beam, multi-beam, beam forming, and stealth state, etc.

\subsection{Communication Capabilities of RIS}

At present, the research hotspots of RIS application in wireless networks include signal enhancement, enhanced coverage, signal neutralization, simultaneous wireless information and power transfer (SWIPT), secure communication, etc. [28].

\section{Signal Enhancement}

In the "weak coverage" and "ultra-distance coverage" areas of the cellular network, the wireless signal of the cellular base station is very weak and often cannot meet the signal-to-noise ratio (SNR) threshold requirement of the receiver to demodulate the signal 
correctly. As the working frequency bands of 5G/B5G/6G are getting higher and higher, the problems of "weak coverage" and "ultra-distance coverage" will become more serious.

The location of RIS deployment should not be too far away from the BS in order to ensure the signal-energy incident on the RIS surface is strong enough. At the same time, this distance should not be too close; otherwise, the diversity gain will be very low. Assuming that only two communication paths - the direct link from BS to the receiver and the indirect link via the RIS as a relay-are considered, since the indirect link can usually be expressed as a cascade channel [17], the received signal is expressed as:

$$
y=\left(\rho e^{-j \mu}+\alpha \beta \gamma e^{-j(\theta+\varphi)}\right) \sqrt{p_{t}} x+n
$$

where, $\rho, \alpha, \beta$ are the channel amplitudes of the direct link from the BS to the receiver, the link from the BS to the RIS, and the link from the RIS to the receiver, respectively; $\mu, \theta, \varphi$ are the corresponding phases; and $\gamma$ is the reflection coefficient of the RIS.

Signal power is usually characterized by the square of the signal amplitude, so the SNR of the received signal can be expressed as:

$$
\eta=\frac{\left|\rho e^{-j \mu}+\alpha \beta \gamma e^{-j(\theta+\varphi)}\right|^{2} p_{t}}{N_{0}}
$$

where, $N_{0}$ denotes the noise power.

In the case of RIS total reflection, the SNR can be maximized if and only if the phase of the signal received through the RIS cascade channel is exactly the same as that received through the direct link. This is because the two path signals can then be coherently summed to maximize the energy of the received signal, thus achieving the maximum data-transmission rate.

Therefore, the best reflection coefficient is:

$$
\gamma^{*}=e^{j(\theta+\varphi-\mu)}
$$

Assuming that the transmission power is determined and the influence of small-scale fading is not considered, the value of SNR is mainly affected by the RIS reflection coefficient. When the RIS-reflected beam is pointed in the same direction as the direct link, SNR can reach its maximum, as follows:

$$
\eta_{\max }=\frac{(\rho+\alpha \beta)^{2} p_{t}}{N_{0}}
$$

Therefore, in signal-enhancement applications, the key is to control the reflected beam phase of the RIS and form as narrow a beam as possible to the receiver. The usual solution is to reversely solve the coding sequence of all units of the RIS through the desired beam pattern according to beamforming theory [29]. Limited by current technical implementation and cost, RIS is not suitable for fast and complex calculations. In fact, the optimal reflection coefficient of RIS can be calculated at the BS and transmitted to the intelligent RIS controller through the dedicated feedback link between BS and RIS. The intelligent controller generates the RIS coding sequences accordingly, which are used as the input signals to control each artificial unit of RIS, so as to form the desired beams.

\section{Coverage Enhancement}

The blocking effect of buildings on high-frequency signals is more obvious in urban areas with tall buildings, especially in large cities. Although operators can deploy more 5G/6G base stations, there will inevitably be many "blind spots" on the back of buildings. Similarly, with the improvement of indoor application requirements, the indoor propagation of high-frequency signals has also received widespread attention. It is not difficult to imagine that indoor walls, rooms, corridors, etc., can seriously hinder the propagation of 
high-frequency signals. Therefore, a large number of low-cost RIS unites can be deployed in suitable locations, such as exterior walls, indoor walls, and corridor corners of buildings in order to change the signal-propagation direction and enhance 5G/6G network coverage.

When the direct link between the base station and the user is completely blocked by a building, the beam of the base station should be directed to RIS units so that the signal energy of the indirect link is as strong as possible. In this case, the received signal energy is mainly determined by the indirect link. As long as the incident beam of the base station is aligned with RIS, and RIS generates the reflected beam aimed at the user with the maximum reflection coefficient, the maximum SNR can be obtained for the receiver [15,17]. Therefore, it is necessary to shape the base station and RIS joint beam to achieve a better "blind-filling" effect.

\section{Signal Neutralization}

In order to improve the system capacity and spectrum efficiency, 4G/5G adopts tighter co-frequency multiplexing strategies, making it easy to generate co-frequency interference, especially in the case of multi-user/multi-cell applications. By adjusting the phase of the reflected signal of the RIS, the received useful signal energy can be enhanced. Similarly, by adjusting the phase of the RIS-reflected signal, the energy of the interference signal can also be reduced, and the adverse effects caused by the interference can be greatly reduced.

RIS can be deployed at the boundaries of multiple cells. For edge users, adjusting the phase of the reflected wave of the interference-signal incident on the RIS to completely reverse the phase of the interference signal that the user directly receives from the interfering base station can cancel the interference-signal energy. In this case, the minimum SNR value of the interfering signal that can be achieved is [28]:

$$
\eta_{\text {min }}=\left\{\begin{array}{cl}
0, & \text { if } \rho \leq \alpha \beta \\
\frac{(\rho-\alpha \beta)^{2} p_{t}}{N_{0}}, & \text { if } \rho>\alpha \beta
\end{array}\right.
$$

For the first scenario, the best reflection coefficient of RIS is $\gamma^{*}=\frac{\rho}{\alpha \beta} e^{j(\theta+\varphi-\mu+\pi)}$. For the second scenario, the optimal value is $\gamma^{*}=e^{j(\theta+\varphi-\mu+\pi)}$.

\section{SWIPT}

Simultaneous wireless information and power transfer (SWIPT) technology simultaneously carries information and energy by radio frequency to receive information and energy simultaneously. There are two types of users in SWIPT: information users and energy users. In future large-scale IoTs applications, SWIPT technology will have broad application prospects. A large number of IoT terminals will be able obtain energy from wireless signals, and the reduction in IoT energy consumption will promote the development of IoT applications.

However, due to severe wireless-signal attenuation, especially in millimeter-wave and $\mathrm{THz}$-frequency bands, the energy that can be collected may be very limited. Using the signal-enhancement capability of RIS, the signal energy can be enhanced at the same time. The performance indicator of data users is SINR, and the noise power is generally relatively small. For data users, although the received signal power is relatively small (about $-100 \mathrm{dBW}$ ), as long as the value of SINR is relatively large, the received signal can be decoded correctly. However, for energy-harvesting users, such as sensors, the minimum received-power requirement is generally above $-40 \mathrm{dBW}$, which is much more than the minimum power required for data users.

\section{Physical-Layer Security Communication}

Millimeter-wave and terahertz communications can provide richer bandwidths and higher data rates, but high-frequency signals are very prone to producing blocking effects, and reliable communication links cannot be established. Therefore, wireless links are vulnerable to security threats. Physical-layer security (PLS) technology has received extensive attention because it can avoid complex high-level key agreement exchanges, thereby 
reducing communication delays and system complexity. There are two types of users in a PLS network: legitimate users and eavesdroppers. The purpose of PLS technology is to maximize the physical-layer-security transmission rate while avoiding interception of information via eavesdropping. At present, physical-layer security communication based on RIS has become an important application of RIS in wireless communication. Utilizing the signal-neutralization capability of RIS can destructively increase the signal energy of legitimate users being eavesdropped on by eavesdroppers. At the same time, using the signal-enhancement capability of RIS can positively increase the signal energy received by legal users. Therefore, the security-transmission rate of the physical layer can be greatly improved.

\section{An Innovative Infrastructure Based on Shape-Adaptive RIS for Smart Industrial IoTs}

This paper proposes an innovative infrastructure based on shape-adaptive RIS suitable for smart industrial IoTs applications, as shown in Figure 3. As 5G has the inherent characteristics of a large number of connections and low latency in support of the IoTs, it is an ideal choice of communication-network architecture for building a modern industrial IoT. With the enhancement of the ability of B5G and 6G to support ultra-high bandwidth, ultra-low latency, and other requirements, as well as the ability of $6 \mathrm{G}$ to support space-airsea-ground integrated communications, B5G and 6G will play a more active role in future industrial IoTs applications.

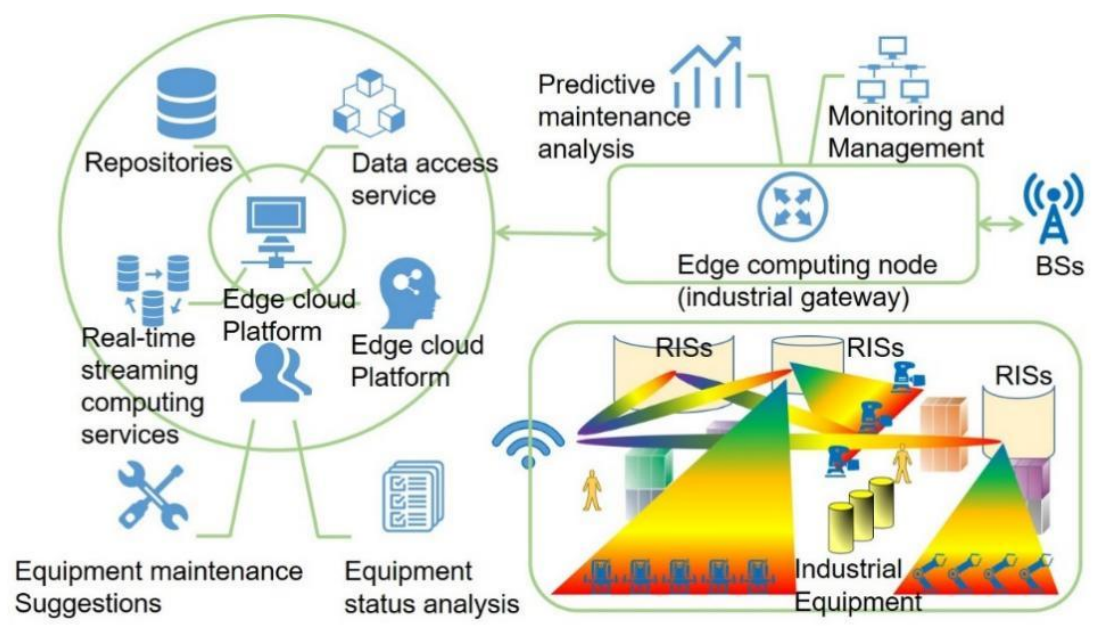

Figure 3. Shape-adaptive RIS-based innovative infrastructure for the intelligent industrial IoTs.

Because $5 \mathrm{G}$ has a higher working frequency band than traditional cellular mobile networks, signal-propagation loss is very serious, leading to a very high demand for $5 \mathrm{G}$ base stations, which will have very high energy consumption and are very likely to cause blind areas or weak coverage areas. This problem will get worse in the B5G and 6G era. Therefore, we propose the RIS infrastructure presented in Figure 3 to enhance the coverage of the industrial IoTs.

In industrial production environments, such as factories and workshops, due to the obstruction of buildings and the existence of various equipment noises, the useful signal power received by IoT terminals will be weak, and the noise power will be relatively strong, thus affecting the normal communication of IoT terminals. We can deploy a large number of RIS units in building corners, workshop partition walls, and areas with a large number of IoT terminals in order to provide RIS-assisted 5G/B5G/6G mobile communication services for the industrial IoTs. In order to adapt to the complex communication environment in the industrial IoTs and to support the communication of aerial IoTs terminals, such as small drones, the shape of the RIS is designed to be mechanically changed. Compared with shape-fixed RIS, shape-adaptive RIS can perform more accurate beamforming, with fewer requirements of the RIS controller, making it easier to implement. In order to reduce 
the implementation cost of RIS, the shape of RIS can be changed mechanically rather than electrically in order better meet the large demand for RIS in industrial IoT scenarios.

By deploying a large number of RIS units in industrial IoTs scenarios and designing RIS to adaptively change shape mechanically according to the conditions of the communication environment, the reflective surface of RIS can be directed toward IoT terminals, thereby greatly increasing the data-transmission rate. At the same time, without adding more mobile base stations, this mechanism can also greatly expand the coverage of wireless signals and achieve deep coverage in factories and workshops. Utilizing the SWIPT capability, RIS can provide energy transmission for industrial IoT terminals. Moreover, by aiming RIS units at legitimate IoT terminals rather than intruder devices, the probability of intruders obtaining useful signals can be greatly reduced, which fundamentally provides a security guarantee for industrial IoT communications.

In order to provide QoS-guaranteed secure connections for industrial applications, edge-computing devices can be deployed at the edge of the industrial IoTs to provide edge intelligent services nearby, as shown in Figure 3. For example, in the application of real-time intelligent detection of industrial equipment, edge-computing devices obtain real-time images taken by high-definition cameras at industrial sites and perform real-time analysis based on artificial intelligence algorithm models to obtain optimal decisions for industrial applications and meet the detection requirements of diversified products. The cloud platform receives aggregated data information from edge-computing devices, performs model training, and pushes the updated model to the edge to optimize detection accuracy and meet the key requirements of complex and diverse industrial IoT application scenarios.

\section{Shape-Adaptive RIS}

\subsection{Shape Design of Deformable RIS}

Currently, RIS regulation is mainly carried out in spatial, time, and frequency domains [30]. The goal of spatial-domain regulation is mainly to control the propagation behavior of reflected waves in space by designing the arrangement of RIS artificial units. The purpose of time-domain regulation is to realize real-time adjustment of RIS. It is the key to achieving reconfigurable, programmable and adaptive RIS. At its core is an intelligent controller based on FPGA, which can write a program composed of multiple coding schemes on FPGA to control various functions in real time. The aim of frequency-domain regulation is actually to control the spectral characteristics of the RIS-reflected wave. By controlling the phase and amplitude of the RIS metasurface units to periodically change according to a certain rule, the spectrum of the incident wave can be expanded on the RISreflected wave, thereby tapping some potential of RIS in terms of spectral characteristics.

Either way, the aim of RIS is essentially to achieve independent control of the phase of each artificial unit. However, in some cases, it is hoped that RIS can achieve flexible phase adjustment in wider ranges, even requiring it to reach 360 degrees, i.e., all phases are adjustable. This is a relatively stringent requirement for RIS, which is difficult to achieve through conventional regulations. Therefore, in this paper, we propose a shape-adaptive RIS mechanism to regulate RIS-reflected waves in the shape domain. The mechanism can reduce the requirements of the RIS controller and artificial unit design, expand the spatial distribution of reflected beams, and achieve high-quality and deep coverage of wireless signals.

The shape-adaptive RIS mechanism constitutes a new type of fractal structural units. Our proposed design of RIS artificial units is based on Minkowski fractal structure [31] in the case of 2bit quantization, as shown in Figure 4. This structured RIS distinguishes the phase of units through the difference in the size of the transmission line, so as to achieve a high-precision RIS beamforming. The greater the quantization accuracy, the greater the accuracy of the characterizable phase, the more accurate the direction of beamforming, and the more concentrated main-lobe energy. Of course, the problem is that the design and control of the units are more complicated. 


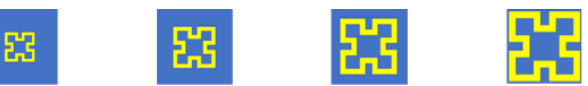

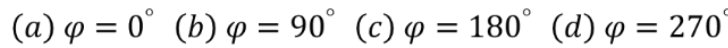

Figure 4. RIS artificial units based on Minkowski fractal structure.

As shown in Figure 5, the RIS metasurface is composed of a large number of scalable artificial units, and different transmission-line sizes of the units correspond to different reflection phases. The base station obtains the UE location information from the UE's uplink-access signal and calculates the direction of the RIS-reflected wave. The FPGA controller reversely solves the phases of each unit of the RIS metasurface according to the desired beam pattern and obtains the desired reflection phase by controlling the size of the transmission line of each unit, thereby realizing the expected beamforming. This shape-adaptive RIS is realized by flexibly adjusting the size of the transmission line of each unit in real time and has special requirements for the selection of electronic-device types, the location of electronic devices, and the unit design of the fractal structure.

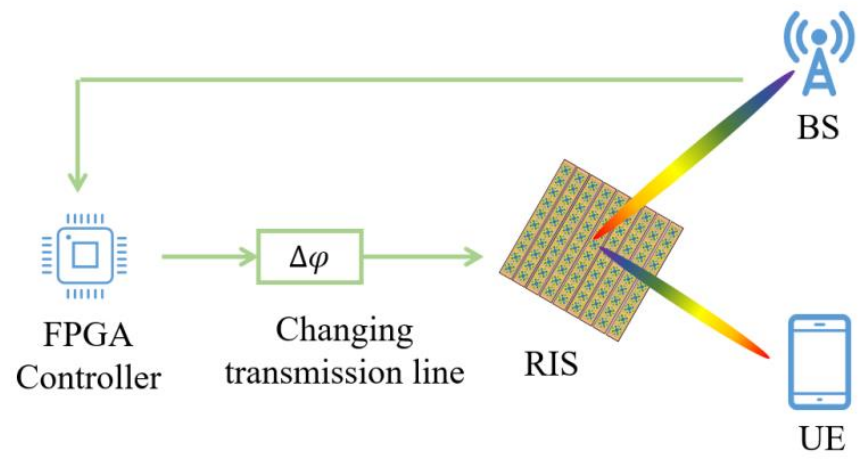

Figure 5. Shape-adaptive RIS with scalable unit sizes.

In fact, without changing the shape of the RIS metasurface, the ability to adjust the direction of the RIS-reflected beam through phase control is limited. However, by designing a deformable RIS, a precise direction of the RIS-reflected beam can be easily achieved. As shown in Figure 6a, when the UE moves outside the coverage of the RIS-reflected signal, the shape of the RIS is adaptively transformed in the form of a cylindrical-patch antenna array [32]. The deformed RIS metasurface points to the current location of the UE, which can continuously provide high-quality communication services for the UE. As shown in Figure $6 \mathrm{~b}$, if the RIS metasurface is designed in a hemispherical form, the RIS-reflected beam can be directed to the air, thereby extending the ground base-station signal to the higher airspace, enabling communication with air UEs, such as drones.

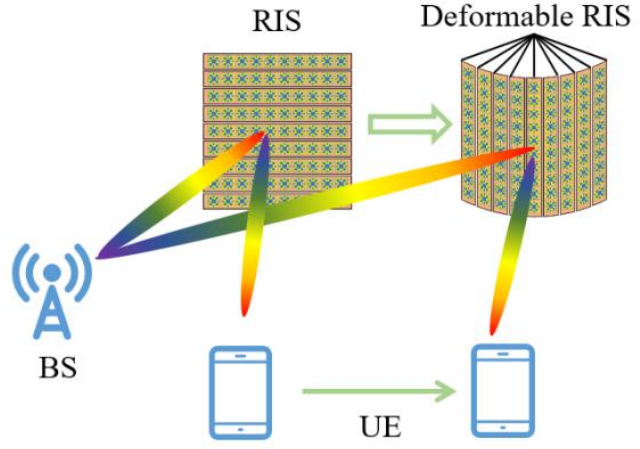

(a)

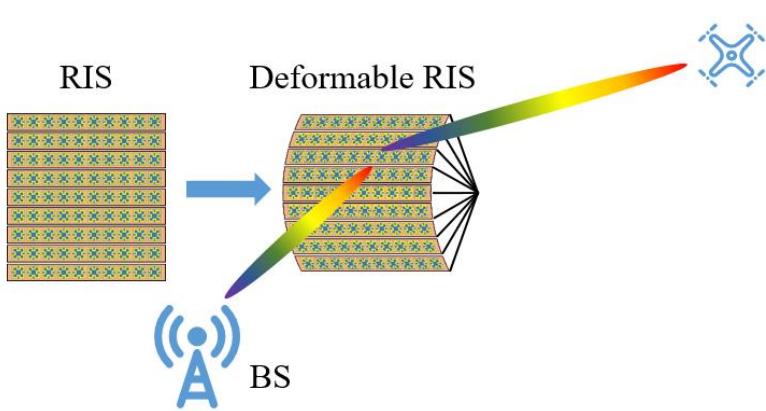

(b)

Figure 6. (a) Deformable RIS supporting the movement of ground UEs. (b) Deformable RIS supporting the communication of air UEs (e.g., drones). 
Shape-adaptive RIS can also realize multi-band applications of information metamaterials, which is very important in current communication systems. The $5 \mathrm{G}$ frequency band is actually backward-compatible, and it covers 4G, 3G, 2G and even 1G bands. Moreover, $5 \mathrm{G}$ bands may vary by region. Therefore, when designing information metamaterials, it is not wise to only apply them to a communication system of a certain operating band. It is necessary to realize information metamaterials that support multiple frequency bands through adaptive RIS.

\subsection{Channel Gain}

Channel gain can be used to characterize the performance of a wireless transmission system. Let $P_{t}$ and $P_{r}$ represent the transmission power and receive power, respectively. Channel gain is defined as:

$$
G=\frac{P_{r}}{P_{t}}
$$

Considering the wireless channel between BS and RIS, it is assumed that the reflection surface of RIS consists of $\sqrt{N}$ rows and $\sqrt{N}$ columns of passive reflection units, and each reflection unit is a small square with length $a$. Then, the area of each unit is $a^{2}$, and the total physical area of the RIS reflecting surface is $N a^{2}$.

As shown in Figure 7, suppose that the BS transmitting antenna is centered in front of the RIS surface, and the distance is $d_{0}$. On the RIS two-dimensional plane, the distance between a reflection unit and the center is $\sqrt{x^{2}+y^{2}}$. The distance from the BS to the reflection unit is:

$$
d=\sqrt{d_{0}^{2}+x^{2}+y^{2}}
$$

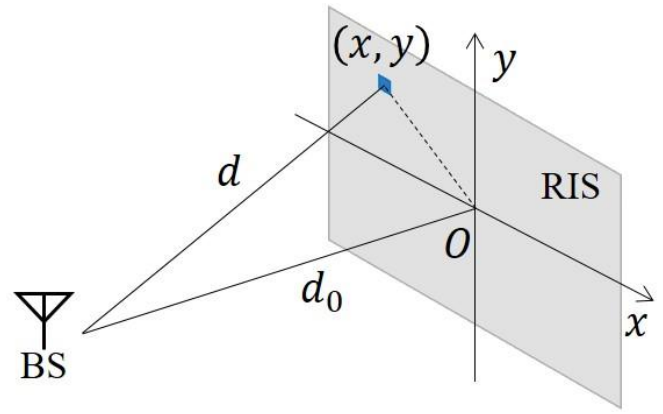

Figure 7. The distance from BS to RIS-reflection units.

Without considering the effects of BS antenna gain and Rayleigh fading, for spherical waves emitted by BS, channel gain is directly related to the propagation distance and the area of the receiving antenna. Therefore, the channel gain from the BS to each RIS reflection unit is:

$$
g=\frac{a^{2}}{4 \pi d^{2}}
$$

For a shape-adaptive RIS antenna array, assuming that the direction and polarization of each reflecting unit perfectly match the incident wave from the BS, the total channel gain is:

$$
G_{\text {deformable-RIS }}=N g
$$

Since RIS is passive, Equation (9) must satisfy $0<G_{\text {deformable }-R I S} \leq 1$, i.e., $N a^{2} \leq 4 \pi d^{2}$. In other words, the reflection ability of RIS cannot be continuously enhanced by blindly increasing the area of RIS reflection surface.

However, for general RIS with a planar array, the direction and polarization of each reflecting unit cannot always perfectly match the incident wave. Therefore, the effective 
receiving area of the RIS will not always be equal to the actual RIS physical area. According to the literature [33], the total channel gain in this case is, approximately:

$$
G_{\text {with }-R I S}=\frac{N g_{0}}{3\left(N g_{0} \pi+1\right) \sqrt{2 N g_{0} \pi+1}}+\frac{2}{3 \pi} \tan ^{-1}\left(\frac{N g_{0} \pi}{\sqrt{2 N g_{0} \pi+1}}\right)
$$

\section{Simulation Results and Analysis}

Assuming that the direct links between the BS and the UEs are completely blocked, communication is completely achieved through the indirect link provided by the RIS. Since RIS is passive, it does not introduce new interference. Therefore, the interference at the receiver only considers the multiple-access interference caused by other UEs. Suppose that the RIS has $N$ passive reflection units, and each UE is configured with one antenna unit; fading from these reflection units to the UE is unrelated.

If the receiver's normal working threshold is $\eta_{0}$, the cumulative distribution function (CDF) of SNR is:

$$
F\left(\eta_{0}\right)=P\left(\eta \leq \eta_{0}\right)
$$

It can be seen that $F\left(\eta_{0}\right)$ represents the probability of communication interruption. The probability that the system can communicate normally, i.e., the coverage probability, is $1-F\left(\eta_{0}\right)$.

In order to compare the changes in the coverage probability caused by the use of deformable RIS, we performed the simulations in Figure 8. The simulation parameters were that the operating frequency band of the system is $3.5 \mathrm{GHz}$, the BS is equipped with an $8 \times 8 \mathrm{MIMO}$ antenna, the antenna height is $30 \mathrm{~m}$, the downtilt angle is $15^{\circ}$, and the transmission power is $50 \mathrm{dBm}$. One RIS is deployed on the ceiling of a factory workshop with a height of $10 \mathrm{~m}$, which has $16 \times 16$ reflective units with intervals and sizes of $\lambda / 2$. The antenna downward tilt angle of the RIS is $0^{\circ}$. There are 10 single-antenna IoT terminals in the workshop, all with a height of $1.5 \mathrm{~m}$.

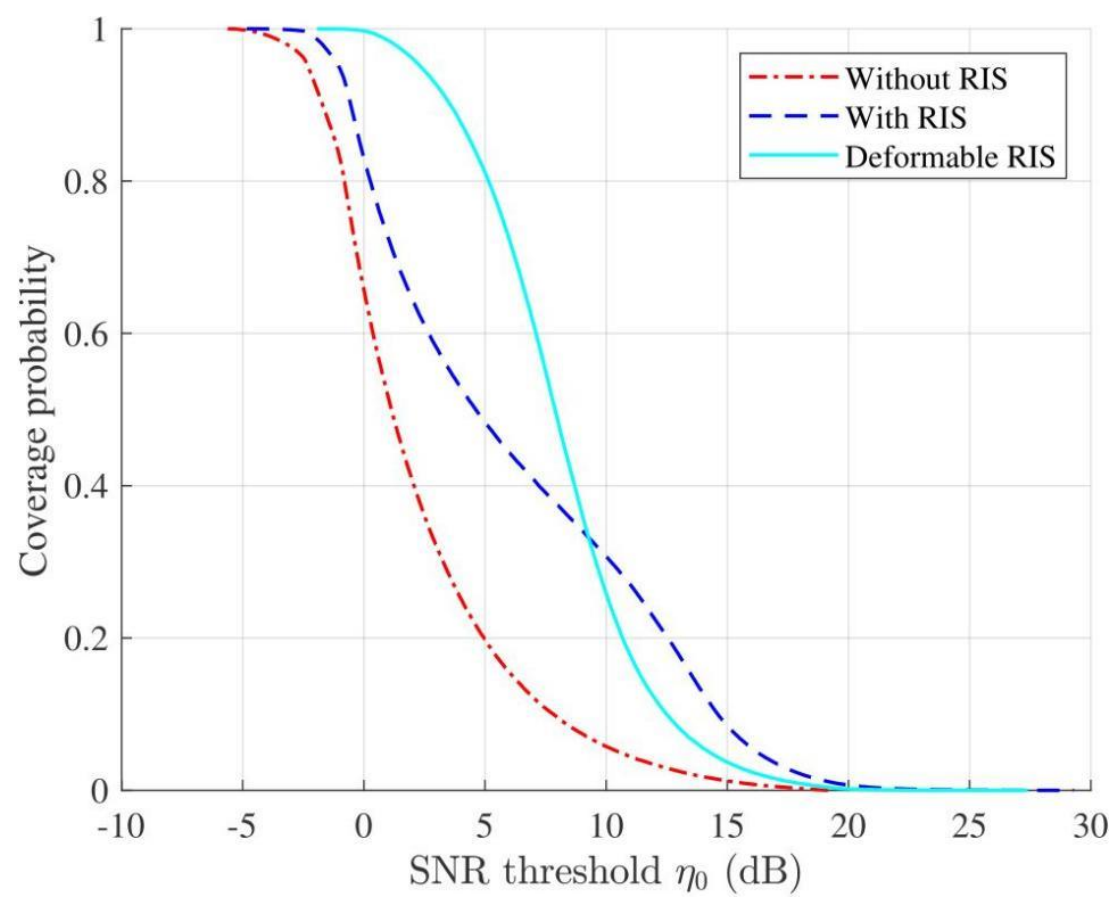

Figure 8. Coverage probability in different situations.

Figure 8 shows that as the SNR threshold increases, the coverage probability of the system drops rapidly until it cannot meet the normal communication requirements. However, under the normal SNR threshold, the RIS-assisted communication systems can 
successfully provide communication services for more IoT terminals, and the coverage probability is better than that of a general communication system. Moreover, because the shape-adaptive RIS can cover a larger area, its system performance is the best.

Figure 9 shows the channel gain of the wireless channel between BS-RIS under theoretical analysis. As RIS is passive and does not have a signal-amplification function, when the number of reflection units is small, the channel gain is very weak, as can be seen in Figure 9. However, as the number of units increases, the channel gain increases approximately linearly until a corresponding constant value is reached. When the number of reflective units is small, the channel gain of the deformable RIS is basically the same as that of the general RIS with a planar array. However, when the number of reflective units is large enough, the channel gain of the former is better than that of the latter, which shows the performance of deformable RIS is related to its surface size. It can also be seen from Figure 9 that the path loss caused by the long-distance transmission of wireless signals can be compensated for by increasing the RIS surface area.

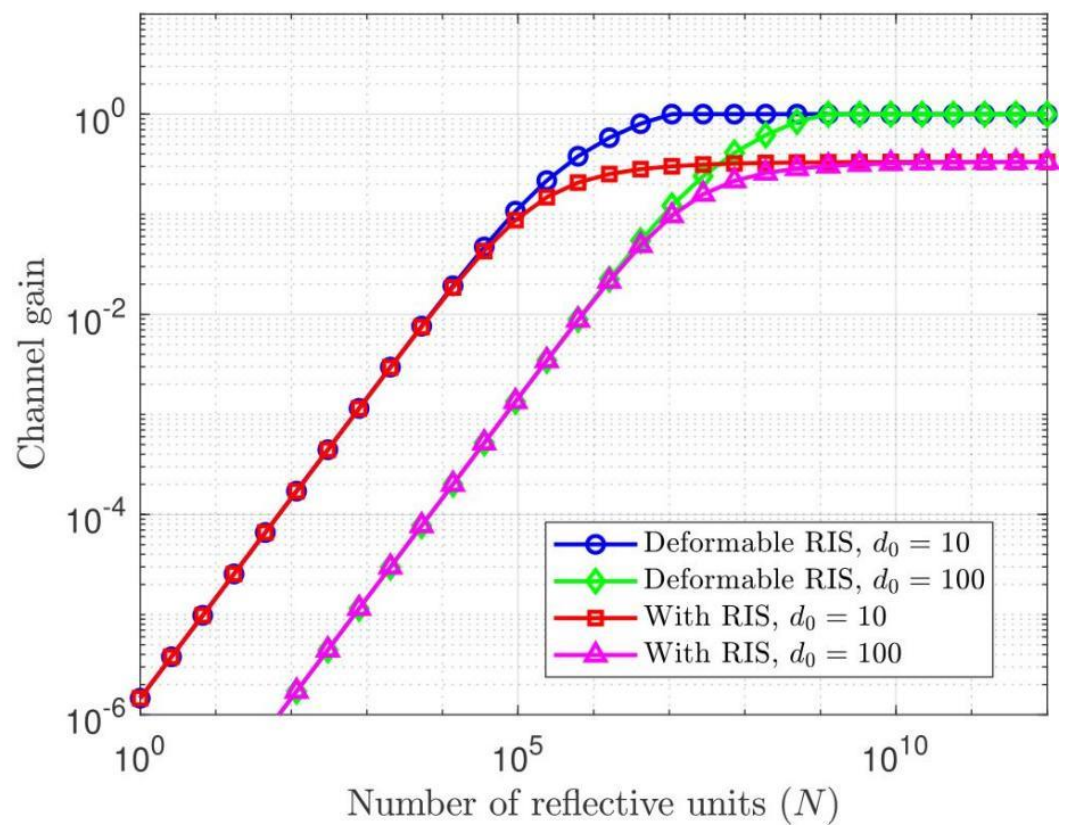

Figure 9. Channel gain of the wireless channel between BS and RIS.

From the above analysis, it can be concluded that the proposed shape-adaptive RIS can provide better system performance in terms of system coverage and transmission rate. Since the channel gain is directly related to the number of RIS reflective units, the metasurface area should be considered when designing the hardware structure of shape-adaptive RIS. If the surface area is too small, the additional channel gain brought by shape adaptation will not be obtained. On the other hand, if it is too large, production cost will be wasted due to reaching the limit of channel gain. Although the complexity of the hardware design of shape-adaptive RIS may be slightly greater than that of general RIS architecture, the former is easier to implement and less expensive to produce in general because it greatly reduces the need for algorithm design and computational processing resources. Moreover, shape-adaptive RIS can facilitate the development of industrial IoT applications due to its ease of deployment since it shape is more adaptive to the surface of the deployed objects.

\section{Conclusions}

In recent years, many researchers have investigated the system functions of RIS, such as signal enhancement, signal neutralization, simultaneous wireless information and power transfer technology, physical-layer security communication, and other capabilities of RIS. The industrial IoT is expected to be a major application scenario for RIS in the future, and 
RIS will aid in the realization of various new applications in the industrial IoTs, such as digital twins (DTs) [34,35]. DTs will play a pivotal role in the future development of IoT due to their successful application and good development prospects in aerospace and smart-manufacturing fields. DTs applications are mainly composed of physical entities (i.e., physical products) and virtual entities (i.e., digital products) and the dynamic data connections between them. Since most of physical products in the IoTs will be wirelessly connected to the Internet, the dynamic data connections between physical entities and virtual entities imply huge demands for wireless channel capacity and wireless transmission performance. RIS is well suited to meet these demands and help to realize DTs applications.

This paper discusses the application of RIS in the industrial IoTs and presents an innovative infrastructure based on shape-adaptive RIS suitable for the intelligent industrial IoTs. The proposed network architecture can achieve deep coverage in factories and workshops, enhance the coverage of wireless signals, and greatly increase the data-transmission rate. At the same time, by deploying edge-computing nodes and edge cloud platforms at the edge of the industrial IoTs, it can also provide intelligent and real-time services for future industrial IoTs applications. As part of our investigation, the SINR and channel gain of RIS were simulated. The simulation results show that the RIS-assisted wireless communication system can achieve better performance, as measured by SINR, than a general wireless communication system. Moreover, because the shape-adaptive RIS employs more flexible beamforming methods, its system performance is better. The simulation results, measured by the channel gain, also illustrate this point.

At present, research on RIS hardware in industry is still at the prototype stage, and there is a lack of complete test platforms and prototype systems. Therefore, research on RIS-based wireless communication systems is basically based on software simulations. Nevertheless, a large amount of RIS research, especially in the area of baseband algorithms, has proven that RIS can be very helpful in improving the performance of wireless transmission environments. As the maturity of RIS materials and devices, as well as resolution of deployment and location issues, the industrialization process of RIS will be greatly promoted. This study provides a typical use case for industrial application of RIS. In the future, more RIS-based wireless network architectures and applications will be proposed.

Author Contributions: J.W. and X.F. conceived, designed and wrote the initial draft of the paper. J.W. did the simulation work, and R.P. and M.K. read and revised the manuscript. All authors have read and agreed to the published version of the manuscript.

Funding: This work was supported by the Open Foundation of State Key Laboratory of Networking and Switching Technology (Beijing University of Posts and Telecommunications) (Funding No. SKLNST-2020-1-10).

Data Availability Statement: The data in this paper is obtained by MATLAB simulation.

Acknowledgments: We thank the State Key Laboratory of Networking and Switching Technology for the financial support.

Conflicts of Interest: The authors declare no conflict of interest.

\section{References}

1. Coutinho, R.; Boukerche, A. Modeling and Analysis of a Shared Edge Caching System for Connected Cars and Industrial IoT-Based Applications. IEEE Trans. Ind. Inform. 2020, 16, 2003-2012. [CrossRef]

2. Horejsi, P.; Novikov, K.; Simon, M. A Smart Factory in a Smart City: Virtual and Augmented Reality in a Smart Assembly Line. IEEE Access 2020. [CrossRef]

3. Elangovan, E. Industry 5.0: The Future of the Industrial Economy, 1st ed.; CRC Press: Boca Raton, FL, USA, 2022; ISBN 978-1-00319067-7.

4. Sardar, S.K.; Sarkar, B.; Kim, B. Integrating Machine Learning, Radio Frequency Identification, and Consignment Policy for Reducing Unreliability in Smart Supply Chain Management. Processes 2021, 9, 247. [CrossRef]

5. Ullah, M.; Sarkar, B. Recovery-Channel Selection in a Hybrid Manufacturing-Remanufacturing Production Model with RFID and Product Quality. Int. J. Prod. Econ. 2020, 219, 360-374. [CrossRef] 
6. Chatzimisios, P.; Soldani, D.; Jamalipour, A.; Manzalini, A.; Das, S.K. Special Issue on 6G Wireless Systems. J. Commun. Netw. 2020, 22, 440-443. [CrossRef]

7. Tariq, F.; Khandaker, M.; Wong, K.K.; Imran, M.A.; Debbah, M. A Speculative Study on 6G. IEEE Wirel. Commun. 2020, 27, 118-125. [CrossRef]

8. Cui, T.-J.; Wu, H.-T.; Liu, S. Research progress of information metamaterials. Acta Phys. Sin. 2020, 69, 158101-158113. [CrossRef]

9. $\mathrm{Wu}, \mathrm{Q} . ;$ Commun. Mag. 2020, 58, 106-112. [CrossRef]

10. Tang, W.; Chen, M.Z.; Chen, X.; Dai, J.Y.; Cui, T.J. Wireless Communications with Reconfigurable Intelligent Surface: Path Loss Modeling and Experimental Measurement. IEEE Trans. Wirel. Commun. 2020, 20, 421-439. [CrossRef]

11. Dai, J.Y.; Tang, W.; Yang, L.X.; Li, X.; Chen, M.Z.; Ke, J.C.; Cheng, Q.; Jin, S.; Cui, T.J. Realization of Multi-Modulation Schemes for Wireless Communication by Time-Domain Digital Coding Metasurface. IEEE Trans. Antennas Propag. 2020, 68, 1618-1627. [CrossRef]

12. Dai, L.; Wang, B.; Wang, M.; Yang, X.; Tan, J.; Bi, S.; Xu, S.; Yang, F.; Chen, Z.; Renzo, M.D.; et al. Reconfigurable Intelligent SurfaceBased Wireless Communications: Antenna Design, Prototyping, and Experimental Results. IEEE Access 2020, 8, 45913-45923. [CrossRef]

13. Pérez-Adán, D.; Fresnedo, Ó.; González-Coma, J.P.; Castedo, L. Intelligent Reflective Surfaces for Wireless Networks: An Overview of Applications, Approached Issues, and Open Problems. Electronics 2021, 10, 2345. [CrossRef]

14. Basar, E.; Di Renzo, M.; De Rosny, J.; Debbah, M.; Alouini, M.-S.; Zhang, R. Wireless Communications Through Reconfigurable Intelligent Surfaces. IEEE Access 2019, 7, 116753-116773. [CrossRef]

15. Björnson, E.; Özdogan, Ö.; Larsson, E.G. Intelligent Reflecting Surface Versus Decode-and-Forward: How Large Surfaces Are Needed to Beat Relaying? IEEE Wirel. Commun. Lett. 2020, 9, 244-248. [CrossRef]

16. Perović, N.S.; Tran, L.-N.; Di Renzo, M.; Flanagan, M.F. Achievable Rate Optimization for MIMO Systems with Reconfigurable Intelligent Surfaces. IEEE Trans. Wirel. Commun. 2021, 20, 3865-3882. [CrossRef]

17. Wu, Q.; Zhang, R. Intelligent Reflecting Surface Enhanced Wireless Network via Joint Active and Passive Beamforming. IEEE Trans. Wirel. Commun. 2019, 18, 5394-5409. [CrossRef]

18. Hong, S.; Pan, C.; Ren, H.; Wang, K.; Nallanathan, A. Robust Transmission Design for Intelligent Reflecting Surface Aided Secure Communication Systems with Imperfect Cascaded CSI. IEEE Trans. Wirel. Commun. 2021, 20, 2487-2501. [CrossRef]

19. Praia, J.; Pavia, J.P.; Souto, N.; Ribeiro, M. Phase Shift Optimization Algorithm for Achievable Rate Maximization in Reconfigurable Intelligent Surface-Assisted THz Communications. Electronics 2022, 11, 18. [CrossRef]

20. Alghamdi, R.; Alhadrami, R.; Alhothali, D.; Almorad, H.; Faisal, A.; Helal, S.; Shalabi, R.; Asfour, R.; Hammad, N.; Shams, A.; et al. Intelligent Surfaces for 6G Wireless Networks: A Survey of Optimization and Performance Analysis Techniques. IEEE Access 2020, 8, 202795-202818. [CrossRef]

21. Björnson, E.; Sanguinetti, L.; Wymeersch, H.; Hoydis, J.; Marzetta, T.L. Massive MIMO Is a Reality-What Is next?: Five Promising Research Directions for Antenna Arrays. Digit. Signal Process. 2019, 94, 3-20. [CrossRef]

22. Chen, Z.; Ma, X.; Han, C.; Wen, Q. Towards Intelligent Reflecting Surface Empowered 6G Terahertz Communications: A Survey. China Commun. 2021, 18, 93-119. [CrossRef]

23. Guo, X.; Chen, Y.; Wang, Y. Learning-Based Robust and Secure Transmission for Reconfigurable Intelligent Surface Aided Millimeter Wave UAV Communications. IEEE Wirel. Commun. Lett. 2021, 10, 1795-1799. [CrossRef]

24. Zhang, M.; Tan, L.; Huang, K.; You, L. On the Trade-Off between Energy Efficiency and Spectral Efficiency in RIS-Aided Multi-User MISO Downlink. Electronics 2021, 10, 1307. [CrossRef]

25. Esfahani, A.; Mantas, G.; Matischek, R.; Saghezchi, F.B.; Rodriguez, J.; Bicaku, A.; Bastos, J. Lightweight Authentication Mechanism for M2M Communications in Industrial IoT Environment. IEEE Internet Things J. 2019, 6, 288-296. [CrossRef]

26. Haochi, Z.; Peihang, H.; Lingyun, N.; Lepeng, Z.; Tiejun, C. Spoof Plasmonic Metamaterials. Acta Opt. Sin. 2021, 41, 0124001. [CrossRef]

27. Lin, B.A.I.; Xin'ge, Z.; Weixiang, J.; Tiejun, C.U.I. Research Progress of Light-controlled Electromagnetic Metamaterials. LDXB 2021, 10, 240-258. [CrossRef]

28. Pan, C.; Ren, H.; Wang, K.; Kolb, J.F.; Elkashlan, M.; Chen, M.; Hanzo, L. Reconfigurable Intelligent Surfaces for 6G Systems: Principles, Applications, and Research Directions. IEEE Commun. Mag. 2021, 59, 14-20. [CrossRef]

29. Cui, T.J.; Mei, Q.Q.; Wan, X.; Jie, Z.; Qiang, C. Coding Metamaterials, Digital Metamaterials and Programming Metamaterials. Light Sci. Appl. 2014, 3, e218. [CrossRef]

30. Huanhuan, Y.; Xiangyu, C.; Jun, G.; Tong, L.I.; Sijia, L.I.; Lili, C.; Xia, Z. Recent Advances in Reconfigurable Metasurfaces and Their Applications. LDXB 2021, 10, 206-219. [CrossRef]

31. Gao, L.H.; Cheng, Q.; Yang, J.; Ma, S.J.; Zhao, J.; Liu, S.; Chen, H.B.; He, Q.; Jiang, W.X.; Ma, H.F. Broadband Diffusion of Terahertz Waves by Multi-Bit Coding Metasurfaces. Light Sci. Appl. 2015, 4, e324. [CrossRef]

32. Hong, H.; Zhao, J.; Hong, T.; Tang, T. Radar-Communication Integration for 6G Massive IoT Services. IEEE Internet Things J. 2021. [CrossRef]

33. Bjrnson, E.; Sanguinetti, L. Power Scaling Laws and Near-Field Behaviors of Massive MIMO and Intelligent Reflecting Surfaces. IEEE Open J. Commun. Soc. 2020, 1, 1306-1324. [CrossRef] 
34. Singh, S.K.; Jeong, Y.S.; Park, J.H. A Deep Learning-Based IoT-Oriented Infrastructure for Secure Smart City. Sustain. Cities Soc. 2020, 60, 102252. [CrossRef]

35. Sepasgozar, S. Differentiating Digital Twin from Digital Shadow: Elucidating a Paradigm Shift to Expedite a Smart, Sustainable Built Environment. Buildings 2021, 11, 151. [CrossRef] 